\title{
Ablação por cateter: uma nova opção de tratamento da Fibrilação Atrial Crônica
}

\author{
Catheter ablation: a new treatment option for Chronic Atrial Fibrillation
}

\begin{abstract}
Ablación con catéter: una nueva opción de tratamiento para la Fibrilación Auricular Crónica
\end{abstract}

Estela Fernandes Pimenta Oliveira ${ }^{1 *}$, Lays Cristina Oliveira Campos ${ }^{1}$, Larissa Lemos Pires de Melo1, Ana Gabriela Souza Rocha ${ }^{1}$, Danielle Teles de Lima1, Lara Braga Martins ${ }^{1}$, Larissa Ungarelli Borges ${ }^{1}$, Lara Juliana Silva de Rezende ${ }^{1}$, Sílvia Avalos Hallak¹.

\section{RESUMO}

Objetivo: Descrever as principais características da fibrilação atrial crônica e apresentar a ablação por cateter como alternativa terapêutica efetiva. Detalhamento do caso: Trata-se de um relato de caso de um paciente com aumento de átrio esquerdo e fibrilação atrial (FA) crônica de difícil controle, que após ser submetido ao tratamento medicamentoso otimizado e posterior cardioversão elétrica sem sucesso, obteve resposta eficiente com ablação por cateter após estudo eletrofisiológico intracardíaco (EEF) e consequente resolução do quadro de FA. O EEF é um método diagnóstico e terapêutico de cateterismo cardíaco, no qual é possível identificar o circuito responsável pela taquicardia e bloquear o foco por meio de uma lesão térmica discreta provocada pelo próprio cateter já inserido, proporcionando a eliminação dos estímulos impulsionadores da arritmia. Considerações finais: $O$ trabalho expõe esta nova alternativa terapêutica, bem como suas vantagens em relação aos tratamentos convencionais já consagrados e a possibilidade de um maior benefício no prognóstico do paciente com FA de difícil controle.

Palavras-chave: Fibrilação atrial, Ablação, Cateterismo cardíaco, Estudo eletrofisiológico.

\section{ABSTRACT}

Objective: To describe the main characteristics of chronic atrial fibrillation and to present catheter ablation as an effective therapeutic alternative. Detail of the case: This is a case report of a patient with enlarged left atrium and chronic AF with difficult to control, who after undergoing optimized drug treatment and subsequent unsuccessful electrical cardioversion, obtained an efficient response with catheter ablation of intracardiac electrophysiological study (EPS) and consequent resolution of AF. EPS is a form of cardiac catheterization, whereby the circuit responsible for tachycardia can be identified and the focus blocked by means of a discrete thermal injury caused by the catheter itself already inserted, providing the elimination of the stimulating of the arrhythmia. Final considerations: The work exposes this new therapeutic alternative, as well as its advantages in relation to conventional treatments already established and the possibility of a greater benefit in the prognosis of patients with AF that are difficult to control.

Descriptors: Atrial fibrillation, Ablation, Cardiac catheterization, Electrophysiological study.

\section{RESUMEN}

Objetivo: Describir las principales características de la fibrilación auricular crónica y presentar la ablación con catéter como una alternativa terapéutica eficaz. Detalle del caso: Se trata de un caso clínico de un paciente con agrandamiento de la aurícula izquierda y fibrilación auricular (FA) crónica de difícil control, que tras un tratamiento farmacológico óptimo y una posterior cardioversión eléctrica infructuosa, obtuvo una

${ }^{1}$ Faculdade de Medicina de Franca (UNIFRAN), Franca - SP. *E-mail: estelafpimenta@gmail.com 
eficaz respuesta con ablación con catéter tras estudio electrofisiológico intracardíaco (EEP) y consecuente resolución de la FA. EEF es un método diagnóstico y terapéutico de cateterismo cardíaco, en el que es posible identificar el circuito responsable de la taquicardia y bloquear el foco mediante una discreta lesión térmica provocada por el propio catéter ya insertado, proporcionando la eliminación de los estímulos estimulantes de la arritmia. Consideraciones finales: El trabajo expone esta nueva alternativa terapéutica, así como sus ventajas en relación a los tratamientos convencionales ya establecidos y la posibilidad de un mayor beneficio en el pronóstico de los pacientes con FA de difícil control.

Palabras clave: Fibrilación auricular, Ablación, Cateterización cardíaca, Estudio electrofisiológico.

\section{INTRODUÇÃO}

A Fibrilação Atrial (FA) é uma arritmia supraventricular com estimulação atrial desordenada e caótica, devido a diferentes focos de despolarização e uma frequência atrial maior do que 400 estímulos por minuto (SOSA E, et al., 2016; FERNANDES JN, et al., 2018). Há um automatismo focal associado a diversos circuitos de reentrada podendo ocorrer áreas de fibrose com períodos refratários atriais encurtados, gerando um ciclo devido à diminuição da complacência atrial (ZIMERMAN LI, et al., 2009).

A FA é apontada como a arritmia sustentada mais comum na prática clínica, sendo estimada uma prevalência que possa chegar a $1 \%$ da população. No entanto, acredita-se que esses valores possam ser ainda maiores, visto a parcela relevante de casos assintomáticos (10 a $25 \%$ ), o que dificulta a procura profissional e consequentemente seu diagnóstico (ZIMERMAN LI, et al., 2009).

Tal estimulação atrial desordenada pode ocorrer em um evento único ou então se apresentar de forma paroxística ou persistente. Há ainda outras classificações quanto a sua frequência e localização, sendo elas: FA recorrente, não valvar e solitária ou isolada. Não obstante tais classificações dependem, respectivamente, de número de episódios prévios, ausência de concomitância com valvopatias e cardiopatias estruturais, pulmonares e hipertensão (MAGALHÃES LP, et al., 2016).

A maioria dos pacientes apresenta-se assintomático. Quando sintomáticos, apresentam-se com palpitações ou irregularidade do pulso. Muitas vezes, apresentam como manifestação inicial eventos tromboembólicos sistêmicos súbitos, visto que a $F A$ representa a maior fonte emboligênica de origem cardíaca conhecida (ZIMERMAN LI, et al., 2009; MAGALHÃES LP, et al., 2016).

Devido a esse grande potencial emboligênico, os portadores de FA têm o risco tromboembólico calculado, visando de forma individual avaliar a necessidade de terapia anticoagulante (SOSA E, et al., 2016).

A correção do ritmo e controle da frequência cardíaca inclui a prática regular de atividades físicas, medidas dietéticas, controle de peso. Medicações específicas de administração via oral ou endovenosa e até mesmo cardioversão de acordo com as necessidades do paciente (FERNANDES JN, et al., 2018).

Quando o paciente apresenta uma FA recorrente, de difícil controle, não apresentando boa resposta com o tratamento medicamentoso otimizado ou anseia uma via de tratamento mais definitiva; a ablação dos focos arrítmicos deve ser considerada. Nos últimos anos a técnica de ablação passou de uma proposta de difícil realização, no início dos anos 90, para um método utilizado com maior constância e sucesso. Contudo, o emprego da ablação deve respeitar normas técnicas específicas e seguir recomendações para a escolha do paciente, a fim de melhores resultados (LINK MS, et al., 2016).

Neste contexto, os estudos eletrofisiológicos (EEF) intracardíacos, são procedimentos realizados pela introdução de cateteres por meio de punção de um vaso (venoso ou arterial) e posicionamento destes cateteres em áreas estratégicas do coração, com o intuito de mapear o processo de despolarização cardíaca daquele paciente e identificar possíveis focos originários da arritmia em questão (SOSA E, et al., 2016; LINK MS, et al., 2016). 
A ablação dos focos arrítmicos por cateter pode ser realizada na ocasião do próprio estudo eletrofisiológico (EEF) intracardíaco ou em uma segunda abordagem. Essa ablação consiste no bloqueio dos focos causadores da arritmia mediante lesões térmicas em áreas estratégicas, provocadas por energia de radiofrequência estabelecida na extremidade do próprio cateter inserido (SASA EA, et al., 1995; PIRES LM, et al., 2013).

Dessa forma, o estudo visa discutir as principais terapêuticas em caso de FA crônica, bem como suas indicações.

\section{DETALHAMENTO DO CASO}

Paciente, sexo masculino, 60 anos, hipertenso mal controlado, até então com seguimento ambulatorial irregular, compareceu ao consultório médico de Cardiologia em setembro de 2014, procurando assistência para controle adequado dos níveis pressóricos. Hipertenso há 25 anos, em tratamento irregular há 12 anos com Losartana $100 \mathrm{mg} / \mathrm{dia}$ e Hidroclorotiazida $25 \mathrm{mg} /$ dia.

Além disso, dislipidêmico em uso de Sinvastatina $40 \mathrm{mg}$, etilista social e sedentário. Sem antecedentes de tabagismo. Histórico familiar positivo para Hipertensão Arterial Sistêmica (HAS) e Acidente Vascular Encefálico (AVE) (Quadro 1).

Quadro 1 - Resumo dos valores do Ecocardiograma do paciente.

\begin{tabular}{|c|}
\hline Dimensões \\
\hline Aorta (AO): 38,2 \\
\hline Átrio esquerdo (AE): 39,8 \\
\hline Ventrículo esquerdo (VE): 45,2 \\
\hline Ventrículo direito (VD): 42,2 \\
\hline Septo interventricular (SIV): 11,6 \\
\hline Parede Posterior: 11,1 \\
\hline D\%: 42\% \\
\hline Fração de ejeção (FE): 73 \\
\hline
\end{tabular}

Fonte: Oliveira EFP, et al., 2020.

Após análise do ecocardiograma do paciente, é possível concluir que há discreta hipertrofia de ventrículo esquerdo (HVE) e Insuficiência Mitral leve. Realizado ajuste medicamentoso, o paciente seguiu com retornos irregulares a cada 6-12 meses, mantendo em seu exame clínico ritmo cardíaco regular, Pressão Arterial Sistólica (PAS) entre 140-150 mmHg e Pressão Arterial Diastólica (PAD) em torno de 90mmHg. Iniciado em uso de aparelho de pressão positiva contínua (Continuous Positive Airway Pressure - CPAP) em 2017 devido a quadro de apneia do sono.

Em março de 2018, foi diagnosticada fibrilação atrial após paciente retornar ao ambulatório para consulta de rotina, assintomático. Realizado novo Ecocardiograma que evidenciou cardiopatia hipertensiva com Átrio esquerdo 48 (anterior de 39,8) e, então, iniciado anticoagulante Rivaroxabana 20 mg e Amiodarona 200 $\mathrm{mg} / \mathrm{dia}$. Solicitado Holter não realizado pelo paciente.

Em maio de 2018, devido a persistência do quadro de fibrilação atrial, após Ecocardiograma Transesofágico não evidenciar trombo intracardíaco, foi internado e realizado Amiodarona endovenosa (EV) e duas cardioversões elétricas bem-sucedidas, recebendo alta em ritmo sinusal, que voltou a se tornar irregular após decorridos 3 dias do procedimento.

Então, o paciente foi encaminhado ao estudo eletrofisiológico (EEF). Ressalta-de que no período de uso de amiodarona, paciente desenvolveu hipotireoidismo como efeito colateral do medicamento. No estudo eletrofisiológico $(E E F)$, foi realizado isolamento elétrico das veias pulmonares (bloqueio de entrada de veias pulmonares) e ablação em istmo cavotricúspideo. O paciente foi cardiovertido com sucesso, mantendo-se então em ritmo sinusal. 
Retornou ao ambulatório para seguimento, em novembro de 2018, assintomático, uso regular das medicações e com bom controle dos níveis pressóricos. Ao exame, apresentou-se com permanência do ritmo cardíaco regular, evidenciando o bom resultado da ablação realizada no EEF. Mantida anticoagulação com Rivaroxabana, iniciado processo de diminuição da dose da Amiodarona nesta ocasião e suspensa 30 dias após, por manutenção do ritmo sinusal. Holter evidenciando ritmo sinusal mantido. Atualmente em uso de: Losartana $50 \mathrm{mg}$ de 12/12 h, Bisoprolol $5 \mathrm{mg}$ ao dia, Hidroclorotiazida $25 \mathrm{mg}$ ao dia com bom controle pressórico. Holter: mantendo-se em ritmo sinusal.

\section{DISCUSSÃO}

Por conceito, a FA é uma arritmia que resulta de mecanismos de micro reentradas, inibindo a ação do nó sinusal e gerando ao eletrocardiograma (ECG) um tremor na linha de base, com a medida do intervalo RR irregular. Essa arritmia supraventricular pode ser classificada em Paroxística, Persistente ou Permanente (SOSA E, et al., 2016; FERNANDES JN, et al., 2018).

Dentre a classificação temporal das FA, pode-se destacar que a FA Paroxística é aquela de duração até 07 dias, podendo apresentar resolução espontânea ou resolução após intervenção. Nesse sentido, a FA Persistente apresenta-se em episódios com mais de sete dias de evolução e a FA permanente é caracterizada por quadros onde a fibrilação atrial não foi revertida. (FERNANDES JN, et al., 2018; ZIMERMAN LI, et al., 2009).

Os fatores de risco clássicos envolvidos no desenvolvimento da Fibrilação Atrial (FA) são bastante comuns na população geral, o que significa que grande parcela pode estar predisposta ao desenvolvimento desta arritmia embora não tenha ciência disso. Dentre os fatores de risco associados podem ser citados Hipertensão Arterial Sistêmica (HAS), obesidade, sedentarismo, etilismo, fatores genéticos, doenças valvares, Infarto Agudo do Miocárdio (IAM) e Insuficiência Cardíaca (IC) (MAGALHÃES LP, el al., 2016).

O paciente em questão apresentava-se portador de fatores de risco para o desenvolvimento de Fibrilação Atrial (FA), tais como Hipertensão mal controlada e sedentarismo, condições que evoluíram com cardiopatia hipertensiva, evidenciada no ecocardiograma com hipertrofia de ventrículo esquerdo e insuficiência mitral discreta.

Além dos fatores de risco clássicos, tem sido observada importante influência da Apneia Obstrutiva do Sono (AOS) como fator associado à Fibrilação Atrial (FA), e estudos recentes empenham-se em relacionar os mecanismos envolvidos nesse contexto, como níveis altos de dióxido de carbono (CO2), estresse oxidativo e alterações da pressão intratorácica, estimulando o sistema nervoso simpático e culminando em desequilíbrio do sistema eletrofisiológico cardíaco e em diversas comorbidades cardiovasculares (MAGALHÃES LP, et al., 2016 ; ANDRADE FMD, et al., 2016).

O paciente retratado obteve diagnóstico de AOS aproximadamente um ano antes de ser diagnosticada a arritmia descrita, sendo instituído uso de aparelho de pressão positiva contínua (Continuous Positive Airway Pressure - CPAP).

Inclusive alguns estudos priorizam a necessidade de manter AOS estável em pacientes com FA que sejam eleitos a intervenção com ablação para que o sistema elétrico cardíaco tenha menor risco de se desestabilizar após o procedimento, objetivando a manutenção do resultado da ablação (DRAGER LF, et al., 2018; MEHRA R, et al., 2019).

Avaliar o paciente portador de FA, bem como seu conhecimento sobre sua condição e adesão ao tratamento, é de extrema importância clínica para individualização do plano terapêutico destinado a cada paciente. Diante do caso exposto, é possível observar a irregularidade nas consultas por parte do paciente, assim como a baixa adesão ao tratamento medicamentoso. Tais comportamentos são frequentes na prática clínica e acontecem em razão de fatores intrínsecos e extrínsecos do paciente, como, por exemplo, a ausência de sintomatologia, a posologia medicamentosa prescrita, resistência ao tratamento e até o suporte do sistema de saúde para obtenção da medicação (RAPARELLI V, et al., 2017). 
A FA possui um espectro clínico bastante variado e heterogêneo, podendo apresentar-se desde pacientes assintomáticos até quadros de instabilidade hemodinâmica. Dentro das manifestações, destacamse as palpitações, desconforto torácico inespecífico ou sintomas característicos de Insuficiência Cardíaca como fraqueza, dispneia e sensação de fraqueza, frequência cardíaca entre 140 a 160bpm. Porém, o paciente em estudo apresentava-se assintomático e displicente com seu tratamento de HAS e dislipidemia, e além de sintomas, as consequências que a arritmia pode causar são significativas, entre elas temos: acidentes vasculares isquêmicos ou hemorrágicos, insuficiência cardíaca, alterações cognitivas, complicações socioeconômicas e aumento da mortalidade do paciente (MAGALHÃES LP, et al., 2016; LINK MS, et al., 2016).

O paciente em questão não havia apresentado até o momento alguma dessas consequências, no entanto, com a idade e os demais fatores de risco que estava sendo exposto o colocavam como paciente com alto risco cardiovascular. O tratamento padrão para a FA, previsto pela Sociedade Brasileira de Cardiologia, inicia-se com a anticoagulação e controle da frequência e ritmo cardíacos, ambos realizados via oral, ponderando-se o risco de desenvolvimento de eventos tromboembólicos a partir dos fatores de risco do paciente e o risco de sangramento. Além disso, outra estratégia bastante adotada pelos centros de referência brasileiros é a cardioversão elétrica na tentativa de retornar o ritmo cardíaco regular e sinusal do paciente (FERNANDES JN, et al., 2018).

No entanto, ainda uma grande parcela de pacientes não responde bem aos medicamentos e nem mesmo à cardioversão, ou, ainda, retorna ao ritmo irregular poucos dias após, como ilustrado pelo paciente descrito. Nos últimos anos, vem crescendo a popularidade do tratamento da FA com ablação por cateter, demonstrando, assim, superioridade em relação ao tratamento medicamentoso (HAEGELI LM, et al., 2014). Porém, a escolha desse método deve ser discutida entre médico e paciente, reservado às situações em que a sintomatologia seja exuberante ou que o paciente seja refratário às medidas farmacológicas otimizadas e à cardioversão elétrica, posto que se trata de um procedimento invasivo e que envolve riscos consideráveis de complicações (LINK MS, et al., 2016; AMES A, et al., 2006).

$O$ risco emboliogênico inerente à $F A$ é comprovado, mas também está presente na ocasião do próprio procedimento de ablação por cateter. Por outro lado, há também o risco hemorrágico decorrente do uso das medicações anticoagulantes que forem instituídas no tratamento da FA. Este risco hemorrágico está envolvido principalmente aos anticoagulantes antagonistas de vitamina $\mathrm{K}$. Nesse contexto, 0 uso ininterrupto dos novos anticoagulantes orais, como a Rivaroxabana, no período periprocedimento tem-se demonstrado eficaz quanto à prevenção de eventos tromboembólicos, e ao mesmo tempo, mais seguro que o uso de antagonistas de vitamina $\mathrm{K}$, embora apresente maior custo financeiro (MAGALHÃES LP, el al., 2016).

O paciente descrito no trabalho permaneceu sob o emprego de Rivaroxabana, anticoagulante oral direto, antes, durante e ainda por alguns meses após o procedimento, quando o anticoagulante foi finalmente suspenso devido à resolução do quadro de FA com a ablação. Ressalta-se que é importante avaliar individualmente as circunstâncias de cada paciente, calcular seu risco emboliogênico e de sangramento, para que assim seja instituída a melhor alternativa para cada caso submetido ao procedimento (BIANCO I, et al., 2020; CARDOSO R, et al., 2020).

Estudos afirmam que os pacientes com melhores resultados pós ablação são aqueles que apresentam FA paroxística, sem doenças cardíacas adjacentes e átrio esquerdo não dilatado (HAEGELI LM, et al., 2014). Porém, nota-se um aumento considerável de resultados positivos em estudos que consideram candidatos para ablação aqueles com insuficiência cardíaca congestiva ou com diminuição da fração de ejeção (LINK MS, et al., 2016).

No presente estudo, percebe-se que os dados do paciente supracitado corroboram com a literatura quando se trata das indicações formais para indicação de ablação por cateter, visto a recorrência do ritmo de fibrilação atrial mesmo após tratamento com anticoagulantes orais, além de discreto aumento de átrio esquerdo (MAGALHÃES LP, et al., 2016). Nesse sentido, o procedimento realizado no caso descrito foi realizado sem complicações e com resultados sintomatológicos e eletrocardiográficos favoráveis após o procedimento (ODOZYNKI G, et al., 2018). 
Com o intuito de manter o ritmo sinusal em pacientes com FA paroxística e FA persistente, a ablação por cateter surgiu como uma estratégia mais eficaz e está sendo utilizada em todo o mundo (OLIVEIRA F, et al., 2016). Um levantamento realizado pela Sociedade Brasileira de Arritmias mostrou que esse procedimento tem sido mais utilizado em todas regiões do país em comparação ao período inicial de instalação, entre os anos de 1998 e 2001. Além disso, na amostra desse mesmo estudo, 59\% usou a técnica de ablação do istmo cavotricuspídeo, mesmo essa sendo uma técnica mais utilizada para Flutter Atrial, sendo uma técnica eficaz em caso de fibrilação atrial persistente ou resistente as outras opções de tratamento realizadas anteriormente (FENELON G, et al., 2007).

O presente relato evidenciou os fatores de risco, a clínica e as opções terapêuticas da Fibrilação Atrial. Com ele pôde-se observar sua relevância e interferência na vida do paciente, além de identificar que o tratamento medicamentoso dessa condição já está bem estabelecido, mas, que em alguns casos, deve-se pensar na utilização da ablação com cateter como método alternativo, principalmente para aqueles em que há dificuldade de resposta só com o tratamento inicial. Nesse aspecto há a necessidade de se identificar de forma individualizada os pacientes adequados para se tentar essa opção terapêutica no intuito de ter bons resultados e melhora no quadro de indivíduos com FA persistentes. Ainda, percebe-se a necessidade de ampliar o conhecimento acerca desse método terapêutico e permitir a sua maior acessibilidade, para que os profissionais consigam indicar quando necessário essa possibilidade.

\section{REFERÊNCIAS}

1. SOSA E, et al. Arritmias Cardíacas. Livro Clínica Médica do Hospital das Clínicas da Universidade de São Paulo, 2016; 2: 74-414.

2. NETO JF, et al. Fibrilação Atrial. Revista Qualidade Hc, 2018; 183.

3. ZIMERMAN LI, et al. Diretrizes Brasileiras de Fibrilação Atrial. Sociedade Brasileira de Cardiologia. Arq Bras Cardiol, 2009; 92 (6 supl.1): 1-39.

4. MAGALHÃES LP, et al. II Diretrizes Brasileiras de Fibrilação Atrial. Arq Bras Cardiol 2016; 106(4Supl.2):1-22

5. HAISSAGUERRE M, et al. Ablation of atrial fibrillation. AHA Journals, 2016; 134: 339-352.

6. SASA EA, et al. Indicações para Estudos Eletrofisiológicos e Ablação por Cateter de Arritmias Cardíacas. Recomendações do DAEC da SBC. Arquivo Brasileiro de Cardiologia, 1995; 64.

7. PIRES LM, et al. Ablação de Arritmias por Cateter com Mapeamento Eletroanatômico Exclusivo: uma Série de Casos. Arquivo Brasileiro Cardiologia, 2013.

8. ANDRADE FMD, et al. O papel do exercício físico na apneia obstrutiva do sono. Jornal Brasileiro de Pneumologia, 2016.

9. DRAGER LF, et al. Sleep apnea and cardiovascular disease: lessons from recente trials and need for team Science. Circulation. NCBI; 2018; 136(19): 1840-50.

10. MEHRA R. Sleep apnea and the heart. Cleveland Clinic Journal of Medicine, 2019; 86(1): 10-8.

11. RAPARELLI V, et al. Adherence to oral anticoagulant therapy in patients with atrial fibrillation. Focus on nonvitamin $\mathrm{K}$ antagonist oral anticoagulants. Thromb Haemost. 2017; 117(2): 209-218.

12. HAEGELI LM, et al. Catheter ablation of atrial fibrillation: an update. Eur Heart J. 2014; 35(36): 2454-2459.

13. AMES A, et al. Catheter ablation of atrial fibrillation. Circulation.AHA Journals, 2006; 113: 666-8.

14. SBMFC, et al. Fibrilação atrial. Diretriz NHG M79. Segunda revisão parcial, agosto 2013.

15. BIANCO I, et al. Risco de Fibrilação Atrial após Ablação de Flutter Dependente de Istmo Cavo-Tricuspídeo: Vale a Pena Fazer a Ablação da FA Simultaneamente? Arquivo Brasileiro Cardiologia, 2020; 114(5): 775-82.

16. CARDOSO R, etal. Anticoagulantes Orais Diretos Ininterruptos em Ablação por Cateter de Fibrilação Atrial: Pronto para a Prática Clínica. Arquivo Brasileiro Cardiologia, 2020; 114(3): 443-5.

17. ODOZYNKI GF, et al. O Resultado da Ablação por Cateter da Fibrilação Atrial Paroxística Depende da Anatomia das Veias Pulmonares. Arquivo Brasileiro Cardiologia, 2018; 111(6): 824-830

18. OLIVEIRA FG, et al. Avaliação de Realce Tardio das Veias Pulmonares por Ressonância Magnética de 3 Tesla após Ablação de Fibrilação Atrial. Arquivo Brasileiro Cardiologia, 2016; 29(1): 25-27.

19. FENELON G, et al. Ablação da Fibrilação Atrial no Brasil: Resultados do Registro da Sociedade Brasileira de Arritmias Cardíacas. Arquivo Brasileiro Cardiologia 2007; 89(5): 285-289. 